\title{
Asymptotic behavior of coupled Euler-Bernoulli beams with dissipative joint
}

\author{
Kais Ammari *
}

\begin{abstract}
We consider an example of coupled Euler-Bernoulli beams with dissipative joint. We show that the exponential decay property holds for any position of the joint.
\end{abstract}

\section{Introduction}

In this paper we discuss the exponential stability of coupled Euler-Bernoulli beams with pointwise dissipation at the common end.

The equations considered in this paper are:

$$
\begin{gathered}
\partial_{t}^{2} u+\Delta^{2} u+\partial_{t} u(\xi, t) \delta_{\xi}-\partial_{x t}^{2} u(\xi, t) \frac{d \delta_{\xi}}{d x}=0 \quad \text { in } Q, \\
u=\Delta u=0 \quad \text { on } \Sigma \\
u(x, 0)=u^{0}(x), \partial_{t} u(x, 0)=u^{1}(x) \quad \text { in } \Omega,
\end{gathered}
$$

where $Q=\Omega \times(0, \infty), \Omega=(0,1), \Sigma=\{0,1\} \times(0,+\infty), \Delta=\partial_{x}^{2}$ and $\delta_{\xi}$ is the Dirac mass at the point $\xi \in \Omega$.

Equation (1) governs vibrations of coupled Euler-Bernoulli beams with a dissipative joint. The dissipation acts via the bending moments (i.e $\Delta u$ ) which is proportional to the angular velocity $\partial_{x t}^{2} u(\xi, t)$ at the point $\xi$ and by the shear force $\partial_{x}^{3} u(\xi, t)$ which is proportional to the velocity $\partial_{t} u(\xi, t)$ at the joint $\xi$.

As far as we known this type of problem was not studied, in the case of feedbacks acting simultaneously in bending moments and in shear forces. In the case of only one feedback the problem was studied in [8].

Our main result is the exponential stability of the above system for any position $\xi$ of the joint. To do so, we use a method similar to the one in [7],[8] and [6], by proving that the resolvent remains bounded on the imaginary axis. To show this result, we shall verify, following [8], that the transfer function (see [12]), which relates the input to the output, is bounded on the imaginary axis and on the other hand, that our system is stable and detectable.

${ }^{*}$ Centre de Mathématiques Appliquées, UMR CNRS 7641, École Polytechnique, 91128 Palaiseau Cedex, France e-mail: kais@cmapx.polytechnique.fr 
A different method for proving this result is used in [1] where the variable coefficients case is also tackled.

Now if we write a variational formulation of the problem (1)-(3), we notice that (1)-(3) can be reformulated in the following form:

$$
\begin{gathered}
\partial_{t}^{2} u+\Delta^{2} u=0 \quad \text { in } Q_{\xi}, \\
u=\Delta u=0 \quad \text { on } \Sigma, \\
{[u]_{\xi}=\left[\partial_{x} u\right]_{\xi}=0 \quad \text { in }(0, \infty),} \\
{\left[\partial_{x}^{2} u\right]_{\xi}=\partial_{x t}^{2} u(\xi, t) \quad \text { in }(0, \infty),} \\
{\left[\partial_{x}^{3} u\right]_{\xi}=-\partial_{t} u(\xi, t) \quad \text { in }(0, \infty),} \\
u(x, 0)=u^{0}(x), \partial_{t} u(x, 0)=u^{1}(x) \text { in } \Omega,
\end{gathered}
$$

where $Q_{\xi}=(\Omega \backslash \xi) \times(0, \infty)$ and $[f]_{\xi}=f\left(\xi^{+}\right)-f\left(\xi^{-}\right)$.

In order to prove existence and uniqueness of solutions of (1) - (3), it is enough to apply standard semigroup techniques (see [2]). The following holds:

Proposition 1.1 Let

$$
\left(u^{0}, u^{1}\right) \in\left(H^{2}(\Omega) \cap H_{0}^{1}(\Omega)\right) \times L^{2}(\Omega) .
$$

Then the equations (1) - (3) admit a unique solution

$$
u \in C\left([0, \infty), H^{2}(\Omega) \cap H_{0}^{1}(\Omega)\right) \cap C^{1}\left([0, \infty), L^{2}(\Omega)\right) .
$$

Furthermore, if

$$
\left(u^{0}, u^{1}\right) \in\left(H^{4}(\Omega \backslash \xi) \cap\left(H^{2}(\Omega) \cap H_{0}^{1}(\Omega)\right)\right) \times\left(H^{2}(\Omega) \cap H_{0}^{1}(\Omega)\right),
$$

then (1) - (3) admit a unique solution

$$
u \in C\left([0, \infty), H^{4}(\Omega \backslash \xi) \cap\left(H^{2}(\Omega) \cap H_{0}^{1}(\Omega)\right)\right) \cap C^{1}\left([0, \infty), H^{2}(\Omega) \cap H_{0}^{1}(\Omega)\right),
$$

where

$$
H^{4}(\Omega \backslash \xi)=\left\{u \text { such that } u_{\mid(0, \xi)} \in H^{4}(0, \xi), u_{\mid(\xi, 1)} \in H^{4}(\xi, 1)\right\} .
$$

\section{Exponential stability}

Before stating the exponential stability result of our system, we prove a strong stability result of solutions of (1) - (3). More precisely, the following proposition holds:

Proposition 2.1 For any $\xi \in \Omega,\left(u^{0}, u^{1}\right) \in\left(H^{2}(\Omega) \cap H_{0}^{1}(\Omega)\right) \times L^{2}(\Omega)$ the solutions of (1) - (3) satisfy:

$$
\lim _{t \rightarrow \infty} E(t, u)=0
$$

where

$$
E(t, u)=\int_{\Omega}\left\{(\Delta u)^{2}+\left(\partial_{t} u\right)^{2}\right\} d x .
$$


Proof. As the imbedding of the domain of the infinitesimal generator of the evolution equation in the energy space $\left[H^{2}(\Omega) \cap H_{0}^{1}(\Omega)\right] \times L^{2}(\Omega)$ is obviously compact , the strong stability estimate can be obtained by a simple application of Lasalle's invariance principale. This is why we skip here the proof and we refer, for instance, to $[3]$.

Next, we state and prove the main result of the present paper.

Theorem 2.1 For any $\xi \in \Omega$, there exist $C, \gamma>0$ such that for any solution $u$ of (4) - (9) in $C\left([0, \infty), H^{2}(\Omega) \cap H_{0}^{1}(\Omega)\right) \cap C^{1}\left([0, \infty), L^{2}(\Omega)\right)$, we have:

$$
E(t, u) \leq C e^{-\gamma t} E(0, u), t>0 .
$$

The proof of this theorem is based on crucial arguments given by Rebarber in [8] and also by [4],[10] and [11].

Proof. Let the space $X$ be defined by:

$$
X=\begin{gathered}
{\left[H^{2}(\Omega) \cap H_{0}^{1}(\Omega)\right]} \\
\times \\
L^{2}(\Omega)
\end{gathered},
$$

and equipped with the norm:

$$
\|(u, v)\|_{X}=\left(\int_{\Omega}\left\{(\Delta u)^{2}+v^{2}\right\} d x\right)^{1 / 2} .
$$

Let the operator $A_{0}$ be defined on $X$ by:

$$
A_{0}=\left(\begin{array}{cc}
0 & I \\
-\Delta^{2} & 0
\end{array}\right),
$$

with

$$
\begin{aligned}
& D\left(A_{0}\right)=\left\{\left(\begin{array}{l}
u \\
v
\end{array}\right) \in \begin{array}{c}
H^{4}(\Omega \backslash \xi) \cap H^{2}(\Omega) \\
\times \\
H^{2}(\Omega)
\end{array}, u=v=\Delta u=0 \quad \text { on } \partial \Omega,\right. \\
& \left.\left[\frac{d^{3} u}{d x^{3}}\right]_{\xi}=\frac{d v}{d x}(\xi)=0\right\},
\end{aligned}
$$

note that the spectrum of $A_{0}$ is on the imaginary axis.

We also need to introduce the operator $A_{1}$ defined on $X$ by:

$$
A_{1}=\left(\begin{array}{cc}
0 & I \\
-\Delta^{2} & 0
\end{array}\right), x \neq \xi
$$

with

$$
\begin{gathered}
D\left(A_{1}\right)=\left\{\begin{array}{c}
u \\
v
\end{array}\right) \in \begin{array}{c}
H^{4}(\Omega \backslash \xi) \cap H^{2}(\Omega) \\
\times \\
H^{2}(\Omega)
\end{array} \quad, u=v=\Delta u=0, \\
\text { on } \left.\partial \Omega,\left[\frac{d^{3} u}{d x^{3}}\right]_{\xi}=-v(\xi),\left[\frac{d^{2} u}{d x^{2}}\right]_{\xi}=\frac{d v}{d x}(\xi)\right\} .
\end{gathered}
$$


Let $B_{1}, B_{2} \in \mathcal{L}\left(\mathbb{R},\left(D\left(A^{*}\right)\right)^{\prime}\right)$ be two feedback operators given by:

$$
B_{1}=\left(\begin{array}{c}
0 \\
-\delta_{\xi}
\end{array}\right) \text { and } B_{2}=\left(\begin{array}{c}
\delta^{\prime \prime}\left(.-\xi^{+}\right)-\delta^{\prime \prime}\left(.-\xi^{-}\right) \\
0
\end{array}\right),
$$

where $A^{*}$ is the adjoint of $A$ and $\left(D\left(A^{*}\right)\right)^{\prime}$ is the dual of $D\left(A^{*}\right)$ obtained by means of inner product in $L^{2}(\Omega)$.

Since $A_{0}$ is anti-adjoint then for any $(u, v) \in D\left(A_{0}^{*}\right)=D\left(A_{0}\right)$ we have:

$$
B_{1}^{*}\left(\begin{array}{l}
u \\
v
\end{array}\right)=-v(\xi), B_{2}^{*}\left(\begin{array}{l}
u \\
v
\end{array}\right)=\frac{d^{2} u}{d x^{2}}\left(\xi^{+}\right)-\frac{d^{2} u}{d x^{2}}\left(\xi^{-}\right) .
$$

Now we shall introduce the controlled and the observed systems. The controlled system is given by:

$$
\begin{gathered}
\partial_{t}^{2} u+\Delta^{2} u=0 \quad \text { in } Q_{\xi}, \\
u=\Delta u=0 \quad \text { on } \Sigma, \\
{[u]_{\xi}=\left[\partial_{x} u\right]_{\xi}=0 \quad \text { in }(0, \infty),} \\
w_{1}(t)=\left[\partial_{x}^{3} u\right]_{\xi}, w_{2}(t)=\partial_{x t}^{2} u(\xi, t) .
\end{gathered}
$$

The observed system is the system defined by:

$$
\begin{gathered}
\partial_{t}^{2} u+\Delta^{2} u=0 \quad \text { in } Q_{\xi}, \\
u=\Delta u=0 \quad \text { on } \Sigma, \\
{[u]_{\xi}=\left[\partial_{x} u\right]_{\xi}=0 \quad \text { in }(0, \infty),} \\
V_{1}(t)=C_{1} U(t)=-\partial_{t} u(\xi, t), V_{2}(t)=C_{2} U(t)=\left[\partial_{x}^{2} u\right]_{\xi},
\end{gathered}
$$

where $C_{0}=\left(\begin{array}{l}C_{1} \\ C_{2}\end{array}\right)$ is the observation operator, $B_{0}=\left(B_{1}, B_{2}\right)$ is the control operator and

$$
U=\left(\begin{array}{l}
u \\
u^{\prime}
\end{array}\right) .
$$

We also introduce the controlled-observed system $(C, A, B)$ given by:

$$
\begin{aligned}
& U^{\prime}(t)=A_{0} U(t)+B_{1} w_{1}(t)+B_{2} w_{2}(t), \\
& V_{1}(t)=C_{1} U(t), V_{2}(t)=C_{2} U(t) .
\end{aligned}
$$

This system is given by the following schem:

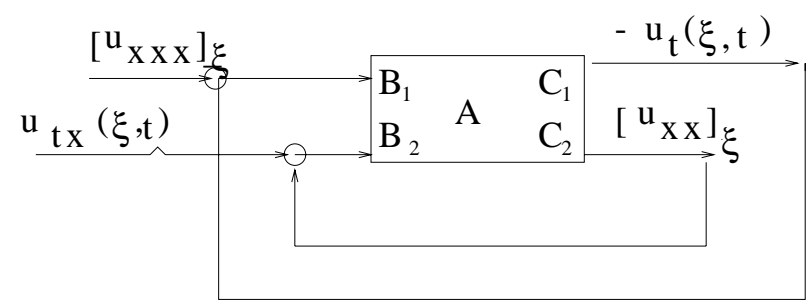

Figure 1: The controlled-observed system 
Theorem 2.2 Assume that the system $(C, A, B)$ is regular, $(A, B)$ is stable and $(C, A)$ is detectable. Then if $G(\lambda)$, the transfert function related to the system $(C, A, B)$, is bounded on $C_{0}^{+}, R(\lambda, A)$ is bounded on $C_{0}^{+}$. This implies that the semigroup generated by $A$ is exponentially stable.

Proof. We refer to [9] for the proof.

Theorem 2.1 is a consequence of the above theorem: since $R\left(\lambda, A_{1}\right)$ is bounded on $C_{0}^{+}=\{z \in \mathbb{C}, \operatorname{Re}(\mathrm{z})>0\}$, then following [6], inequality (10) holds.

Remark 2.1 Theorem 2.2. is used to prove that $R\left(\lambda, A_{1}\right)$ is bounded on $C_{0}^{+}=\{z \in$ $C, \operatorname{Re}(\mathrm{z})>0\}$. Since operator $A_{1}$ is dissipative then it is enough to show that $R\left(\lambda, A_{1}\right)$ is bounded on the imaginary axis.

Before proving that the assumptions in Theorem 2.2 hold for our system we recall some basic definitions concerning regular systems, stable systems, detectable systems and transfer functions.

Definition 2.1 We say that the system $\left(C_{0}, A_{0}, B_{0}\right)$ is regular if:

(1) $\left(B_{i}\right)$ are bounded operators from $\mathbb{R}$ into $X_{-1}$ and satisfy the following condition: there exist $t_{1}, \alpha>0$ such that for any $w_{i} \in L^{2}\left(\left(0, t_{1}\right), \mathbb{R}\right)$, we have:

$$
\left\|\int_{0}^{t_{1}} S(t-\tau) B_{i} w_{i}(\tau) d \tau\right\|_{X} \leq \alpha\left\|w_{i}\right\|_{L^{2}\left(\left(0, t_{1}\right), \mathbb{R}\right)} \text { for } i=1,2 .
$$

$\left(B_{i}\right)$ are then called admissible control operators;

(2) $\left(C_{i}\right)$ are bounded operators from $D\left(A_{0}\right)$ into $\mathbb{R}$ satisfying the following:

there exist $t_{1}, \alpha>0$ such that for any $x \in D\left(A_{0}\right)$, we have:

$$
\left\|C_{i} S(t) x\right\|_{L^{2}\left(\left[0, t_{1}\right), \mathbb{R}\right)} \leq \alpha\|x\|_{X}, i=1,2 .
$$

$\left(C_{i}\right)$ are called admissible observation operators;

(3) the range of $R\left(\lambda, A_{0}\right) B_{0}$ belongs to $D\left(C_{L}\right)$ for some $\lambda \in \rho\left(A_{0}\right)$;

(4) $C_{0 L} R\left(\lambda, A_{0}\right) B_{0}$ is bounded in the half plane

$$
C_{\alpha}^{+}=\{z \in C, \operatorname{Re}(\mathrm{z})>\alpha\}
$$

for some $\alpha \in \mathbb{R}$, where $S(t)=e^{t A_{0}}$ and $C_{0 L}$ is the Lebesgue extension of $C_{0}$

$$
C_{0 L} x=\lim _{\tau \rightarrow 0} \frac{1}{\tau} \int_{0}^{\tau} C_{0} S(t) x d t
$$

for $x \in X$ where the limit exists in $X$,

$X_{-1}$ the closure of $X$ in the norm $\|\cdot\|_{X_{-1}}=\left\|\left(\beta I-A_{0}\right)^{-1} \cdot\right\|_{X}$ for $\beta$ in the resolvent (the definition of the space $X_{-1}$ is independent of $\beta$, see Weiss [13]).

Definition 2.2 $G=\left(G_{i j}\right)_{1 \leq i, j \leq 2}$ is called the transfer function of the system $\left(C_{i}, A_{0}, B_{j}\right)_{1 \leq i, j \leq 2}$ if it is given by the following equation:

$$
\widehat{V}_{i}(\lambda)=G_{i j}^{0}(\lambda) \widehat{w}_{j}(\lambda), i, j=1,2
$$

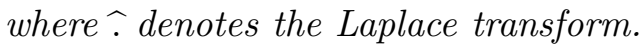


Definition 2.3 The system $\left(A_{0}, B_{0}\right)$ is stable if there exists $F=\left(F_{1}, F_{2}\right)$ such that

(1) the system $\left(F, A_{0}, B_{0}\right)$ is regular;

(2) $\left(I-G_{F}\right)$ is invertible in $H_{\infty}^{\infty}$;

where $G_{H}$ is the transfert function related to the system $\left(F, A_{0}, B_{0}\right), H_{\alpha}^{\infty}$ is the space of anlytical functions bounded on $C_{\alpha}^{+}$(this is a Hilbert space equipped with the norm $\left.L^{\infty}\right)$ and

$$
H_{\infty}^{\infty}=\cup_{\alpha \in \mathbb{R}} H_{\alpha}^{\infty} ;
$$

(3) $A_{0}+B_{0} F$ is the generator of an exponentially stable semigroup of $X$.

Definition 2.4 The system $\left(C_{0}, A_{0}\right)$ is detectable if there exists $H=\left(H_{1}, H_{2}\right)$ such that

(1) $\left(C_{0}, A_{0}, H\right)$ is regular;

(2) $\left(I-G_{H}\right)$ is invertible in $H_{\infty}^{\infty}$;

where $G_{H}$ is the transfert function related to the system $\left(C_{0}, A_{0}, H\right)$,

(3) $A_{0}+H C_{0 L}$ is the generator of an exponentially stable semigroup of $X$ exponentiellement stable.

To verify that our system satisfies the assumptions of Theorem 2.2 we need the following technical result:

Lemma 2.1 (1) Let $G^{1}$ be the transfert function of the following system:

$$
\begin{gathered}
\partial_{t}^{2} u+\Delta^{2} u=0 \quad \text { in } Q_{\xi}, \\
u=\Delta u=0 \quad \text { on } \Sigma, \\
{[u]_{\xi}=\left[\partial_{x} u\right]_{\xi}=0 \quad \text { in }(0, \infty),} \\
\left(\left[\partial_{x}^{3} u\right]_{\xi}+\partial_{t} u(\xi, t),\left[\partial_{x}^{2} u\right]_{\xi}-\partial_{x t}^{2} u(\xi, t)\right)=\left(q_{1}(t), q_{2}(t)\right)=Q(t), \\
C_{0} U(t)=\left(-\partial_{t} u(\xi, t),\left[\partial_{x}^{2} u\right]_{\xi}\right) .
\end{gathered}
$$

Then

$$
\lim _{\lambda \rightarrow \infty} G^{1}(\lambda)=0, \lambda \in \mathbb{R}
$$

(2) $A_{1}=A_{0}-B_{1} B_{1 L}^{*}-B_{2} B_{2 L}^{*}$.

Before proving this lemma we decompose $B_{0}^{*}$ on a basis of eigenvectors of $A_{0}$.

Let $\left(\Phi_{w}\right)_{w}$ be a basis of eigenvectors of $A_{0}$ in $X$,

where

$$
\Phi_{ \pm w}(x)=C_{w}^{-2}\left(\begin{array}{l}
\phi_{w}(x) / \pm i w^{2} \\
\phi_{w}(x)
\end{array}\right)
$$

with

$$
C_{w}^{-2}=\int_{0}^{1}\left\{\left(\frac{\Delta \phi_{w}}{w^{2}}\right)^{2}+\phi_{w}^{2}\right\} d x .
$$

If we decompose the operator $B_{0}^{*}$ we arrive at:

$$
B_{0}^{*}=\left(\sum_{w} b_{w}^{1} \Phi_{w}^{*}, \sum_{w} b_{w}^{2} \Phi_{w}^{*}\right),
$$


where

$$
b_{w}=\left(\begin{array}{c}
b_{w}^{1} \\
b_{w}^{2}
\end{array}\right), b_{ \pm w}=B_{0}^{*} \Phi_{ \pm w}(\xi)=:\left(\begin{array}{c}
B_{1}^{*} \Phi_{ \pm w} \\
B_{2}^{*} \Phi_{ \pm w}
\end{array}\right)
$$

and

$$
\Phi_{w}^{*}=C_{w}^{-2}\left(\phi_{w}(x) / \pm i w^{2}, \phi_{w}(x)\right) .
$$

Therefore, we can represent the operator $B_{0}$ by the following vector of $X \times X$ :

$$
\left[b_{w}^{1}, b_{w}^{2}\right]_{w} .
$$

To compute the coefficients $b_{w}$ we need the following lemma.

Lemma 2.2 The eigenvalues and eigenvectors of the operator $A_{0}$ are given by:

(1) $\lambda=0$ is an eigenvalue of $A_{0}$ with mutiplicity 1 and its associated eigenvector is $\left(\phi_{0}, 0\right)$

where

$$
\begin{aligned}
& \phi_{0}(x)=x^{3}+\left(3 \xi^{2}-6 \xi+2\right) x, x \in[0, \xi), \\
& \phi_{0}(x)=(x-1)^{3}+\left(3 \xi^{2}-1\right)(x-1), x \in(\xi, 1] ;
\end{aligned}
$$

(2) If $\xi$ satisfies $\cos n \pi \xi=0$ for some $n \in \mathbb{Z}$ then $\lambda= \pm i(n \pi)^{2}$ are the eigenvalues of $A_{0}$, which are with multiplicity 1 and their associated eigenvectors are given by:

$$
\Phi_{ \pm n}(x)=\left(\begin{array}{l}
\phi_{n}(x) / \pm i(n \pi)^{2} \\
\phi_{n}(x)
\end{array}\right)
$$

where

$$
\phi_{n}(x)=\sin (n \pi x), x \in[0,1]
$$

(3) Let $w$ be a positive real such that

$$
g(w)=\operatorname{th}(w \xi)-\operatorname{tg}(w \xi)-\operatorname{th}[w(\xi-1)]+\operatorname{tg}[w(\xi-1)]=0 .
$$

Then $\lambda= \pm i w^{2}$ are the eigenvalues of $A_{0}$, which are with multiplicity 1 and their associated eigenvectors are given by:

$$
\Phi_{ \pm w}(x)=\left(\begin{array}{c}
\frac{ \pm i \phi_{w}(x)}{w^{2}\left\{\xi\left(\frac{1}{\cos ^{2}(w \xi)}-\frac{1}{c h^{2}(w \xi)}\right)+(\xi-1)\left(\frac{1}{\operatorname{ch}^{2}[w(1-\xi)]}-\frac{1}{\cos ^{2}[w(1-\xi)]}\right)\right\}^{1 / 2}} \\
\frac{\phi_{w}(x)}{\left\{\xi\left(\frac{1}{\cos ^{2}(w \xi)}-\frac{1}{\operatorname{ch}^{2}(w \xi)}\right)+(\xi-1)\left(\frac{1}{\operatorname{ch}^{2}[w(1-\xi)]}-\frac{1}{\cos ^{2}[w(1-\xi)]}\right)\right\}^{1 / 2}}
\end{array}\right),
$$

where

$$
\begin{aligned}
& \phi_{w}(x)=\frac{\operatorname{sh}(w x)}{\operatorname{ch}(w \xi)}-\frac{\sin (w x)}{\cos (w \xi)}, x \in[0, \xi), \\
& \phi_{w}(x)=\frac{\operatorname{sh}[w(x-1)]}{\operatorname{ch}[w(\xi-1)]}-\frac{\sin [w(x-1)]}{\cos [w(\xi-1)]}, x \in(\xi, 1] .
\end{aligned}
$$

Proof. We refer to [8] for the proof of this lemma. 
Remark 2.2 Let

$$
I=\{w \geq 0 \text { such that } g(w)=0\},
$$

then since $g(w)$ is analytic, I is a countable family. Thus, we denote by

$$
\lambda_{ \pm k}= \pm i w_{k}^{2}, k \in \mathbb{N} \backslash\{0\} \text { and } w_{0}=0 .
$$

Now we are ready to compute the coefficients $\left(b_{w}\right)_{w}$.

If $\lambda=0$ then:

$$
b_{0}=C_{0}^{2}\left(\begin{array}{c}
0 \\
-6
\end{array}\right) \text {. }
$$

If $w \in I \backslash\{0\}$ then:

$$
b_{w}=\left(\begin{array}{c}
\frac{-\frac{s h(w \xi)}{\operatorname{ch}(w \xi)}+\frac{\sin (w \xi)}{\cos (w \xi)}}{\left\{\xi\left(\frac{1}{\cos ^{2}(w \xi)}-\frac{1}{\operatorname{ch}^{2}(w \xi)}\right)+(\xi-1)\left(\frac{1}{\operatorname{ch}^{2}[w(\xi-1)]}-\frac{1}{\cos ^{2}[w(\xi-1)]}\right)\right\}^{1 / 2}} \\
\frac{2 \frac{\operatorname{sh}[w(\xi-1)]}{\operatorname{ch}[w(\xi-1)]}-2 \frac{\operatorname{sh}(w \xi)}{\operatorname{ch}(w \xi)}}{\left\{\xi\left(\frac{1}{\cos ^{2}(w \xi)}-\frac{1}{\operatorname{ch}^{2}(w \xi)}\right)+(\xi-1)\left(\frac{1}{\operatorname{ch}^{2}[w(\xi-1)]}-\frac{1}{\cos ^{2}[w(\xi-1)]}\right)\right\}^{1 / 2}}
\end{array}\right) .
$$

If $\cos (n \pi \xi)=0$ for some $n \in \mathbb{Z}$ then:

$$
b_{ \pm n}=\left(\begin{array}{c}
\mp \sin (n \pi \xi) \\
0
\end{array}\right) .
$$

Note that $b_{ \pm w}= \pm b_{w}$.

Proposition 2.2 The operator $B_{0}$ is an admissible control operator and $B_{0}^{*}$ is an admissible observation operator.

Proof. It is clear from the expression of $b_{w}$ that the sequence $\left(b_{w}\right)_{w}$ is bounded. To conclude that $B_{0}$ is an admissible control operator, we use the Carleson measure criterion (see [5]). This criterion implies in our case (the eigenvalues of $A_{0}$ are on the imaginary axis) that for $h>0$, the number $N_{h}$ of the eigenvalues of $A_{0}$, in the region

$$
\{z \in \mathbb{C}, 0 \leq \operatorname{Re}(\mathrm{z}) \leq \mathrm{h}, \mathrm{a}-\mathrm{h} \leq \operatorname{Im}(\mathrm{z}) \leq \mathrm{a}+\mathrm{h}\}
$$

satisfies

$$
N_{h} \leq M h,
$$

for some $M<\infty$ independent of $a$. Following Lemma 3.6. in [8], for $\xi \in \Omega$, there exist $a, b$ such that:

$$
k \pi+b \leq w_{k} \leq k \pi+a, \forall k \in \mathbb{N} \backslash\{0\} .
$$

It follows that the number of $w_{k}$ which are in intervals of length $M$ is more than $3+\frac{M}{\pi}$, and then the number of eigenvalues of $A_{0}$ in

$$
S_{a, M}=\left\{z \in \mathbb{C}, \operatorname{Re}(\mathrm{z})=0, \mathrm{a}^{2} \leq \operatorname{Im}(\mathrm{z}) \leq(\mathrm{a}+\mathrm{M})^{2}\right\},
$$


is less than $3+\frac{M}{\pi}$. Thus $N_{h} \leq M h$. This implies that $B_{0}$ is an admissible control operator for $S(t)$ and by duality, we obtain that $B_{0}^{*}$ is an admissible observation operator for $S(t)$.

\section{Proof of Lemma 2.1.}

We know that $B_{0}$ can be represented as follows:

$$
B_{0}=\left(\sum_{k} b_{w_{k}}^{1} \Phi_{w_{k}}, \sum_{k} b_{w_{k}}^{2} \Phi_{w_{k}}\right),
$$

and then

$$
R\left(\lambda, A_{0}\right) B_{0}=\left(\sum_{k} \frac{b_{w_{k}}^{1}}{\lambda-\lambda_{k}} \Phi_{w_{k}}, \sum_{k} \frac{b_{w_{k}}^{2}}{\lambda-\lambda_{k}} \Phi_{w_{k}}\right) .
$$

Introduce the following sum:

$$
\left(\frac{b_{0}^{1}}{\lambda}\left(\begin{array}{c}
b_{0}^{1} \\
b_{0}^{2}
\end{array}\right), \frac{b_{0}^{2}}{\lambda}\left(\begin{array}{c}
b_{0}^{1} \\
b_{0}^{2}
\end{array}\right)\right)+\left(\sum_{k \neq 0} \frac{b_{w_{k}}^{1}}{\lambda-\lambda_{k}}\left(\begin{array}{c}
b_{w_{k}}^{1} \\
b_{w_{k}}^{2}
\end{array}\right), \sum_{k \neq 0} \frac{b_{w_{k}}^{2}}{\lambda-\lambda_{k}}\left(\begin{array}{c}
b_{w_{k}}^{1} \\
b_{w_{k}}^{2}
\end{array}\right)\right) .
$$

If $\lambda$ goes to $+\infty$ in the above sum and $\lambda \in \mathbb{R}$, it turns out that the limit is 0 . This gives that the system $\left(B_{0 L}^{*}, A_{0}, B_{0}\right)$ is regular.

According to [8] we have $A_{1}=A_{0}-B_{1} B_{1 L}^{*}-B_{2} B_{2 L}^{*}$.

Proposition 2.3 The system $\left(B_{0 L}^{*}, A_{1}, B_{0}\right)$ satisfies the assumptions of Theorem 2.2 .

Proof. The transfert function of the system $\left(B_{0 L}^{*}, A_{1}, B_{0}\right)$ is bounded on the imaginary axis. In fact, if we consider the following identity:

$$
B_{0 L}^{*}\left(\lambda I-A_{1}\right)^{-1} B_{0}\left(I+B_{0 L}^{*}\left(\lambda I-A_{0}\right)^{-1} B_{0}\right)=B_{0 L}^{*}\left(\lambda I-A_{0}\right)^{-1} B_{0},
$$

then since the transfer function of the system $\left(B_{0 L}^{*}, A_{0}, B_{0}\right)$ which is nothing else than $B_{0 L}^{*}\left(\lambda I-A_{0}\right)^{-1} B_{0}$ is bounded on the imaginary axis and from $\operatorname{Re}\left(B_{0 L}^{*}(\lambda I-\right.$ $\left.\left.A_{0}\right)^{-1} B_{0}\right)=0$, we deduce that the quantity $B_{0 L}^{*}\left(\lambda I-A_{1}\right)^{-1} B_{0}$ is bounded on the imaginary axis and its limit when $\lambda$ goes to infinity with $\lambda \in \mathbb{R}$ is equal to zero (this implies that $\left(B_{0 L}^{*}, A_{1}, B_{0}\right)$ is regular). It remains to show that the system $\left(A_{1}, B_{0}\right)$ is stabilizable and the system $\left(B_{0 L}^{*}, A_{1}\right)$ is detectable. The following technical result holds:

Lemma 2.3 For any $\xi \in \Omega$, there exist $m_{1}, m_{2}>0$ such that:

$$
m_{1}<\left|b_{w_{k}}\right|<m_{2}, \text { for any } k \in \mathbb{N}
$$

where

$$
\left|b_{w_{k}}\right|^{2}=\left|\left(\begin{array}{c}
b_{w_{k}}^{1} \\
b_{w_{k}}^{2}
\end{array}\right)\right|^{2}=\left|b_{w_{k}}^{1}\right|^{2}+\left|b_{w_{k}}^{2}\right|^{2}
$$

Proof. For $\xi \in \Omega$ having the coprime factorization $\frac{p}{q}$, where $q$ is odd, Rebarber proved in [8] that the sequence $\left(b_{w_{k}}^{2}\right)_{k}$ has a lower bound which implies that the 
sequence $\left(b_{w_{k}}\right)_{k}$ has a lower bound. Furthermore, for $\xi \in \Omega$ and $\xi$ having the coprime factorization $\frac{p}{q}$, where $q$ is even, we have:

$$
\left|b_{k}\right|=1 \text {. }
$$

This gives that $\left(b_{k}\right)_{k}$ has a lower bound.

Let $\eta \in(0,1)$, we denote by

$$
A_{\eta}=\left\{k|| \cos \left(w_{k} \xi\right) \mid \geq \eta\right\}
$$

and

$$
B_{\eta}=\left\{k|| \sin \left(w_{k} \xi\right) \mid \geq \sqrt{1-\eta^{2}}\right\} .
$$

So, we shall study the case where $\xi \in \Omega \backslash Q$.

Since we have:

$$
\begin{aligned}
\left|b_{w_{k}}\right|^{2} & =\left|b_{w_{k}}^{1}\right|^{2}+\left|b_{w_{k}}^{2}\right|^{2} \\
& =\frac{\left\{\left(\frac{s h\left(w_{k} \xi\right)}{\operatorname{ch}\left(w_{k} \xi\right)}-\frac{\sin \left(w_{k} \xi\right)}{\cos \left(w_{k} \xi\right)}\right)^{2}+4\left(\frac{\operatorname{sh}\left[w_{k}(1-\xi)\right]}{\operatorname{ch}\left[w_{k}(1-\xi)\right]}+\frac{\operatorname{sh}\left(w_{k} \xi\right)}{\operatorname{ch}\left(w_{k} \xi\right)}\right)^{2}\right\}}{\xi\left(\frac{1}{\cos ^{2}\left(w_{k} \xi\right)}-\frac{1}{\operatorname{ch}^{2}\left(w_{k} \xi\right)}\right)+(\xi-1)\left(\frac{1}{\operatorname{ch}^{2}\left[w_{k}(\xi-1)\right]}-\frac{1}{\cos ^{2}\left[w_{k}(\xi-1)\right]}\right)},
\end{aligned}
$$

and

$$
\left|b_{w_{k}}\right|^{2}=\frac{\left\{\left(\frac{\operatorname{sh}\left[w_{k}(1-\xi)\right]}{\operatorname{ch}\left[w_{k}(1-\xi)\right]}-\frac{\sin \left[w_{k}(1-\xi)\right]}{\cos \left[w_{k}(1-\xi)\right]}\right)^{2}+4\left(\frac{\operatorname{sh}\left[w_{k}(1-\xi)\right]}{\operatorname{ch}\left[w_{k}(1-\xi)\right]}+\frac{s h\left(w_{k} \xi\right)}{\operatorname{ch}\left(w_{k} \xi\right)}\right)^{2}\right\}}{\xi\left(\frac{1}{\cos ^{2}\left(w_{k} \xi\right)}-\frac{1}{\operatorname{ch}^{2}\left(w_{k} \xi\right)}\right)+(\xi-1)\left(\frac{1}{\operatorname{ch}^{2}\left[w_{k}(\xi-1)\right]}-\frac{1}{\cos ^{2}\left[w_{k}(\xi-1)\right]}\right)},
$$

it follows that:

$$
\begin{aligned}
\left|b_{w_{k}}\right|^{2} \geq & \inf \left(\cos ^{2}\left(w_{k} \xi\right), \cos ^{2}\left[w_{k}(1-\xi)\right]\right) \\
& \left\{t^{2}\left(w_{k} \xi\right)+\operatorname{tg}^{2}\left(w_{k} \xi\right)+4 t h^{2}\left[w_{k}(1-\xi)\right]+4 t^{2}\left(w_{k} \xi\right)+\right. \\
& \left.4 \operatorname{th}\left(w_{k} \xi\right)\left(2 \operatorname{th}\left[w_{k}(1-\xi)\right]-\frac{1}{2} \operatorname{tg}\left(w_{k} \xi\right)\right)\right\}
\end{aligned}
$$

and

$$
\begin{aligned}
\left|b_{w_{k}}\right|^{2} \geq & \inf \left(\cos ^{2}\left(w_{k} \xi\right), \cos ^{2}\left[w_{k}(1-\xi)\right]\right) \\
& \left\{t^{2}\left(w_{k} \xi\right)+\operatorname{tg}^{2}\left(w_{k} \xi\right)+4 t h^{2}\left[w_{k}(1-\xi)\right]+4 t^{2}\left(w_{k} \xi\right)+\right. \\
& \left.4 \operatorname{th}\left[w_{k}(1-\xi)\right]\left(2 \operatorname{th}\left(w_{k} \xi\right)-\frac{1}{2} \operatorname{tg}\left[w_{k}(1-\xi)\right]\right)\right\}
\end{aligned}
$$

We consider then two cases:

First case:

$$
\text { if } \operatorname{tg}\left(w_{k} \xi\right) \operatorname{tg}\left[w_{k}(1-\xi)\right]<0,
$$

then according to (27) and (28) we have

$$
\begin{aligned}
\left|b_{w_{k}}\right|^{2} \geq & \inf \left(\cos ^{2}\left(w_{k} \xi\right), \cos ^{2}\left[w_{k}(1-\xi)\right]\right)\left(\frac{1}{2} \operatorname{tg}^{2}\left(w_{k} \xi\right)+\right. \\
& +\frac{1}{2} \operatorname{tg}^{2}\left[w_{k}(1-\xi)\right]+4 t h^{2}\left(w_{k} \xi\right)+4 t h^{2}\left[w_{k}(1-\xi)\right]-\operatorname{th}\left(w_{k} \xi\right) \operatorname{tg}\left(w_{k} \xi\right)-
\end{aligned}
$$




$$
\left.-\operatorname{th}\left[w_{k}(1-\xi)\right] \operatorname{tg}\left[w_{k}(1-\xi)\right]\right)
$$

Which implies according to (24)

$$
\left|b_{w_{k}}\right|^{2} \geq\left\{\begin{array}{l}
\sin ^{2}\left(w_{k} \xi\right), \text { if } \cos ^{2}\left(w_{k} \xi\right)<\cos ^{2}\left[w_{k}(1-\xi)\right] \\
\sin ^{2}\left[w_{k}(1-\xi)\right], \text { else. }
\end{array}\right.
$$

We conclude in this case that $\left(b_{w_{k}}\right)_{k \in B_{\eta}}$ has a lower bound.

Second case:

$$
\text { if } \operatorname{tg}\left(w_{k} \xi\right) \operatorname{tg}\left[w_{k}(1-\xi)\right] \geq 0,
$$

then we obtain according to (24) and (26)

$$
\begin{aligned}
\left|b_{w_{k}}\right|^{2} & \geq \inf \left(\cos ^{2}\left(w_{k} \xi\right), \cos ^{2}\left[w_{k}(1-\xi)\right]\right)\left(\operatorname{tg}^{2}\left(w_{k} \xi\right)+\operatorname{tg}^{2}\left[w_{k}(1-\xi)\right]\right) \\
& \geq\left\{\begin{array}{l}
\sin ^{2}\left[w_{k}(1-\xi)\right], \text { if } \cos ^{2}\left(w_{k} \xi\right)>\cos ^{2}\left[w_{k}(1-\xi)\right] \\
\sin ^{2}\left(w_{k} \xi\right), \text { else. }
\end{array}\right.
\end{aligned}
$$

So, according to (24) we conclude in the two cases that the sequence $\left(b_{w_{k}}\right)_{k \in B_{\eta}}$ has a lower bound. Lemma 3.5 in [8] implies that $\left(b_{w_{k}}\right)_{k \in A_{\eta}}$ has a lower bound. Furthermore, we know that the sequence $\left(b_{w_{k}}\right)_{k}$ is bounded. This gives the claim and ends the proof of the lemma.

\section{End of the proof of Proposition 2.3.}

As a consequence of the fact that the sequence $\left(b_{w_{k}}\right)_{k}$ has an upper and lower bounds is that the system $\left(A_{1}, B_{0}\right)$ is stabilizable and the system $\left(B_{0 L}^{*}, A_{1}\right)$ is detectable (for more details we refer to Lemmas 2.10.,3.10. in [8] ). This completes the proof of Proposition 2.3.

\section{References}

[1] K. Ammari, M. Tucsnak and Z. Liu, Decay rates for a beam with pointwise force and moment feedback, to appear.

[2] K. Ammari, Uniform stabilization of beams by means of pointwise feedbacks, to appear in Diff. and Integral Equations.

[3] C. Dafermos, Asymptotic behavior of solutions of evolution equations in " Nonlinear evolution equations" (M. G. Crandall. Ed. ), pp. 103-123, Academic Press, New York, 1978.

[4] R. F. Curtain and G. Weiss, Wellposedness of triples of operators (in the sense of linear systems theory), in Distributed Parameter Systems,F .Kappel, K. Kunish, and W. Schapacher, eds., Proc. of the Conf. in Vorau, Austria, July 1988, Birkhauser, Basel, 1989.

[5] R.F. Curtain, Admissible input elements for systems in Hilbert space and a Carleson measure criterion, SIAM J. Control Optim. 21 (1983) pp.614-640.

[6] F. Huang, Characteristic conditions for exponential stability of linear dynamical systems in Hilbert space, Ann. Diff Eq, 1(1985), pp. 43-56. 
[7] J. Prüss, On the spectrum of $C_{0}-$ semigroups, Trans. Amer. Math. Soc., 284 (1984), pp. 847-857.

[8] R. Rebarber, Exponential stability of beams with dissipative joints : a frequency domain approach, SIAM J. Control and Optim., 33 (1995), pp.1-28.

[9] R. Rebarber, Conditions for the equivalence of internal and external stability for distibuted parameter systems. IEEE Trans. Autom. Control, Ac-38 (1993), pp.994-998.

[10] D. Salamon, Infinite-dimentional linear systems with unbounded control and observation : A functional analytic approach, Trans. Amer. Math. Soc., 300 (1987), pp. 383-431.

[11] G. Weiss, Admissible observation operators for linear semigroups, Israel J. Math., 65 (1989), pp. 17-43.

[12] G. Weiss, Transfer functions of regular linear systems, part 1 : Characterizations of regularity, Trans. Amer. Math. Soc., 342 (1994), pp. 827-854.

[13] G. Weiss, Admissibility of unbounded operators, SIAM J. Control Optim., 27 (1989), pp.527-545. 